\title{
The Use of Activated Charcoal for Acute Poisonings
}

\author{
Juliana Bonilla-Velez, ${ }^{1,2}$ Darly J. Marin-Cuero. ${ }^{1,2}$
}

\begin{abstract}
Poisoning results from the ingestion of or contact with harmful substances including overdose or incorrect use of any drug or medication. Decontamination measures prevent the absorption of the substance from the gastrointestinal tract to minimize systemic effects. Activated charcoal is the intervention most frequently used in the initial management of patients with acute intoxications. The use of activated charcoal has decreased over time, but there may be a subgroup of patients who would benefit from its use. In this review we describe the epidemiology of intoxications, the composition and pharmacology of activated charcoal, indications and dosing for use of single dose and multiple doses of charcoal, contraindications, complications and a summary for recommended use of this measure based on published studies.
\end{abstract}

Keywords: Charcoal, decontamination, poisoning, toxicology (Source: MeSH-NLM).

\section{Introduction}

The National Center for Health Statistics (NCHS) defines a poisoning episode as "the event resulting from ingestion of or contact with harmful substances including overdose or incorrect use of any drug or medication" (Available from: http://www. cdc.gov/nchs/nhis/injury_poisoning/ip_glossary.htm, updated January 25, 2010, cited September 10, 2016). Acute poisonings are caused by ingestion of different toxic substances that are found in products we use at home, industry and the pharmaceutical sector, these substances can be acids, bases, salts, heavy metals, iodine tincture and others numerous chemicals. Poisonings impact the patient's health through either local or systemic effects. Local effects depend on the substance's specific chemical characteristics and structure. ${ }^{1,2}$ Systemic effects are obtained when the substance is absorbed and reaches the bloodstream to be subsequently distributed to tissues. ${ }^{1,2}$ The systemic effects can be avoided by performing an intervention to prevent the absorption of the substance from the gastrointestinal tract., ${ }^{1,2}$ There are several interventions have been designed to prevent the absorption of toxic substances, including gastric lavage or administration of an adsorbent, which are called techniques of gastrointestinal decontamination. ${ }^{2,3}$ of these, activated charcoal (AC) is the most frequently used gastrointestinal decontamination method in the initial management of patients with acute intoxications. ${ }^{1,2,4}$ Despite intoxications being among the first causes of accidental death, medical toxicology remains a small subspecialty of emergency medicine and knowledge of these topics among physicians in general can be limited. ${ }^{5}$ Here we present a review of the use of activated charcoal for acute poisonings with a practical approach. The MEDLINE, EMBASE and Current Contents/Science Edition databases were searched mainly with the MeSH terms "Charcoal" and free text "Activated Charcoal", "gastrointestinal decontamination" and relevant articles selected based on the title and abstract. Articles were also selected from the referen- ces cited in the articles reviewed.

\section{Epidemiology}

A recent systematic review of data from the World Health Organization, PubMed and Google reports that unintentional ingestion, inhalation or contact with chemicals caused 346,000 deaths $(7,447,000$ disability-adjusted life years) from acute poisonings in 2004, of which approximately $71 \%$ were estimated to be preventable. ${ }^{6}$ National epidemiological sources such as the United States National Center for Health Statistics reported that poisoning was the second leading cause of injury-related death in 2004 and the rate was higher than at any time since 1968.' By 2008 poisoning became the first cause of accidental death in the US. 5 Data from the US National Vital Statistics System mortality report that deaths from unintentional poisoning increased at a rate of $62.5 \%$, with $95 \%$ of these being secondary to drug use, while poisoning by suicide increased by a rate of 10.8\%. ${ }^{8}$ The American Association of Poison Control Centers' (APCC) National Poison Data System (NPDS) report that in 2014 there were 2,165,142 human exposures reported to the poison control centers, most of which were unintentional (79.4\%) and secondary to single-substance exposures. The main intoxication agents in order of frequency were analgesics (11.3\%), cosmetics/personal care products $(7.7 \%)$, household cleaning substances $(7.7 \%)$, sedatives/hypnotics/antipsychotics (5.9\%), and antidepressants (4.4\%); which was equivalent to $51.9 \%$ being secondary to non-pharmaceuticals and $48.1 \%$ to pharmaceuticals. Ingestion was the main route of exposure (83.7\%). The rate of single-substance exposure-related fatal cases was 620 for pharmaceuticals compared with 262 for non-pharmaceuticals ( $70.3 \%$ and $29.7 \%$ respectively), illustrating how exposures to pharmaceuticals had more severe outcomes. Children younger than 6 years accounted for approximately half of all human exposures (47.7\%). Most toxic exposures occurred at a residence $(93.5 \%)$, followed by the workplace $(1.7 \%)$, schools $(1.3 \%)$, health care facilities $(0.3 \%)$, and restaurants or food 
Table 1. Substances in which Clearance is Increased by Multiple Doses of Activated Charcoal

\begin{tabular}{|c|}
\hline Amitriptyline \\
\hline Carbamazepine \\
\hline Cyclosporine \\
\hline Dapsone \\
\hline Dextropropoxyphene \\
\hline Digitoxin \\
Digoxin \\
\hline Disopyramide \\
Nadolol \\
\hline Phenobarbital \\
\hline Phenylbutazone \\
Phenytoin \\
Piroxicam \\
\hline Propoxyfene \\
Quinine \\
\hline Sotalol \\
\hline Theophylline \\
\hline
\end{tabular}

services $(0.2 \%)$. of all the intoxications reported there were $8.01 \%$ that had a clinically moderate or major effect or resulted in death. The use of activated charcoal has declined over time and is currently listed as the fourth most common method of decontamination used, after Dilute/irrigate/wash, Food/snack, or Fresh air $(46,030$ compared to $796,400,179,470$, and 68,722 cases respectively) but it remains to be the first gastrointestinal decontamination intervention employed. ${ }^{4}$

Likewise, the Central Information Security of Chemicals of Colombia reported that in 2011 there were 4442 calls for advice on toxicological emergencies, where $46.4 \%$ were secondary to voluntary intoxication followed by accidental poisonings, and occupational exposure corresponded to $7.2 \%$ corresponding to 1.76 daily emergencies due to exposure to substances in the workplace, mainly pesticides (CISPROQUIM. Colombian Council of Security: Annual Report on Emergencies 2009. Available from: http://ccs.org.co/interna_cispro.php?idnoticia=142 topcacordeon $=8$, updated $2016 \mathrm{Jul}$ 7; cited $2016 \mathrm{Jul} 7$ ). As illustrated, acute intoxications remain an important cause of morbidity and mortality that vary by location, therefore understanding and applying these concepts in relation to gastrointestinal decontamination can have a large impact on the outcome for these patients.

\section{Composition and Mechanism of Action of AC- tivated Charcoal \\ Activated Charcoal}

Activated charcoal is a mixture of particles which are insoluble and are produced by heating pulverized carbonaceous substances (for example sawdust, peat, or coconut shells) to extreme temperatures $\left(600-900^{\circ} \mathrm{C}\right)$. Subsequently this substance is submitted to the "activation phase" by using steam to erode the internal surfaces of the product, this results in an increase of its adsorptive surface area. ${ }^{1,2}$ The average surface area for activated charcoal is 800-1,200 m2/g.1 "Superactivated" charcoal may have a surface area of 2,800-3,500 m2/g and can adsorb greater quantities of the substance..$^{1.9}$

\section{Mechanism of Action}

Activated charcoal acts like an agent that adsorbs substances localized in the gastrointestinal tract and retains them within the charcoal, thus minimizing the absorption into the bloodstream and reducing or preventing systemic toxicity. ${ }^{1,2,10,11}$ The terms adsorption and absorption are therefore used distinctively to describe each process. To ensure contact of the charcoal with the toxic agent prior to absorption by the mucosa it must be administered as soon as possible. Any delay may decrease its effectiveness. ${ }^{1,2}$

The absorptive surface of activated charcoal contains several chemical forms, such as carbonyl and hydroxyl groups, which adsorbs toxic substances with different affinities.' Studies in vitro have indicated that several factors can influence its adsorptive capacity, such as temperature, pore size, particle size, surface area, solubility and toxic ionization stage, the $\mathrm{pH}$, the presence of inorganic salts, and the gastric contents; most of which cannot be controlled or modified when providing clinical care to the intoxicated patient. ${ }^{12}$

The effectiveness of activated charcoal depends on multiple factors: the longer time elapsed from ingestion to administration reduces its efficacy, as well as presence of food in the gastrointestinal tract, inherent chemical composition and characteristics of the substance itself (for example lipid solubility) and the presence of other substances that decrease intestinal transit time. In addition, effectiveness can also be altered due to the phenomenon of desorption, which can occur when a complex of activated charcoal and a toxic weak acid change from a highly acidic $\mathrm{pH}$ in the stomach to an alkaline $\mathrm{pH}$ in the small intestine that leads to release of the substance from the charcoal and thus may lead to some absorption of substance into the body. Some suggest that this phenomenon rarely has clinical significance. ${ }^{1,12}$

Furthermore, volunteer studies demonstrate that the maximum reduction in the absorption of toxic substances was obtained when active charcoal was administered within the first hour of toxic intake. ${ }^{1,13}$ Some studies have shown that activated charcoal can be effective up to 4 hours after ingestion but efficacy decreases over time. ${ }^{13,14}$ Other studies have shown inconsistent results on the benefit of its administration two hours after the ingestion of the toxic substance. ${ }^{1,11}$ Therefore there is a potential benefit of administration of activated charcoal if administered after 1 hour from ingestion. In addition, due to the adsorptive effect and prevention of absorption of toxic substances into the bloodstream, some authors have suggested that $A C$ may enhance the elimination of substances. ${ }^{12-14}$ This will be further discussed in section 4.3.

\section{Indications}

Use of gastrointestinal decontamination measures in the emergency department continues to be a controversial topic in the toxicology literature. ${ }^{14}$ The formal recommendation from the American Academy of Clinical Toxicology and the European Association of Poisons Centres and Clinical Toxicologists in their position paper in 1997 recommended its use only within an 
hour of the intoxication and this recommendation was maintained in their subsequent 2005 update. ${ }^{1,15}$ There has not been much literature published in this topic over the past 2 decades and therefore under the paradigm of evidence-based medicine there is limited data to support the use of activated charcoal, and some authors have suggested that combined with the low mortality observed in intoxicated patients administration may not be warranted. ${ }^{14}$ However, there could be a subgroup of patients who would benefit from activated charcoal and efforts have been made to offer recommendations for patient selection which will be described below.

The two well designed clinical trials evaluating the benefit of activated charcoal had significant limitations. Cooper et al. randomized all patients over 16 who presented within 12 hours of an intoxication to receiving activated charcoal or no decontamination. The study was performed between July 1999 and October 2000 on sequential patients who presented to the Emergency Department at The Canberra Hospital in Australia. Patients were randomized to activated charcoal (AC) or no gastro-intestinal decontamination as indicated by the sealed sequentially numbered envelope contents. The trial recruited 327 patients over 16 months. The most common substances ingested were benzodiazepines, acetaminophen and selective serotonin reuptake inhibitor antidepressants. More than $80 \%$ of patients presented within 4 hours following ingestion. There were no differences between $A C$ and no decontamination in terms of length of stay (AC 6.75 h, IQR 4-14 vs. controls 5.5 h, IQR 3-12; $\mathrm{p}$ 0.11) or secondary outcomes including vomiting, mortality and intensive care admission. ${ }^{16}$ Some of the limitations on this study is that it included patients who were unlikely to develop significant toxicity and would have therefore had good outcomes irrespective of $A C$ and it excluded seven patients with severe toxicity. ${ }^{14}$ The second study by Eddleston et al. who conducted a randomized controlled trial comparing outcomes for patients treated with single or multiple doses of activated charcoal (SDAC and MDAC respectively) to no charcoal (no AC). They report no reduction in risk of death from use of multiple dose activated charcoal, with odds ratio of MDAC vs no AC 0.93 (Cl 95\% 0.69-1.25), SDAC vs no AC 1.05 ( $\mathrm{Cl} 95 \% 0.79-1.40$ ), MDAC vs SDAC 0.89 ( $\mathrm{Cl} 95 \%$ 0.66-1.19). ${ }^{77}$ Unfortunately this study mainly included patients with intoxications from pesticides and oleander so it is difficult to apply these findings to patients who have had intoxications from prescription drugs. ${ }^{14}$ No studies have been performed on selected patients based on strict criteria to attempt to identify the subgroup of patients who would benefit from these interventions. A summary of important studies has been performed by other authors and is available for review. ${ }^{14,18,19}$

\section{Single Dose of Activated Charcoal}

There are no absolute indications for the administration of activated charcoal. ${ }^{2}$ Universal routine administration is not recommended in the poisoned patient. $2,10,11,14,16,17,20$ Activated charcoal should be administered in patients who have a history of ingestion of a potentially toxic amount of a substance and present to an emergency department within the first hour of the event. ${ }^{1,10,11}$ However, the benefit of its administration on patients out of this time-frame cannot be excluded.' The national guideline for acetaminophen overdose in Australia and New Zealand recommend use of $\mathrm{AC}$ up to 2 hours after ingestion. ${ }^{21}$
Greene et al,2 suggest that activated charcoal should be administered when all the following criteria are met:

$\begin{array}{ll}\text { 1. Intake of a potentially toxic substance. } \\ \text { 2. } & \text { No contraindications for its use. }\end{array}$

3. The ingested substance is susceptible to be adsorbed by activated charcoal.

4. There is high probability that the substance is still in the gastrointestinal tract at the time of administration.

5. The patient is alert and can protect its airway, or has been intubated prior to its administration.

6. The gastrointestinal tract is anatomically and functionally intact.

7. There are no alternative therapies that are safer and/ or more effective.

Bailey et al. recommends the implementation of the "Triangle of gastrointestinal decontamination" to determine whether or not to perform gastrointestinal decontamination.22 The triangle can be used to evaluate the risk versus benefit of this therapy and should be applied considering all the variables at the same time and not consecutively. ${ }^{3,22}$

- Will the intake of this substance have significant effects?

- Can gastrointestinal decontamination change the outcome for this patient?

- Can the administration of activated charcoal be deleterious to the patient?

Isbister et al. suggest considering administration of activated charcoal in two groups of patients: One group are cooperative patients where charcoal may reduce length of hospital stay. The other group of patients include those with a life-threatening poisoning that is not easily treated, such as arrhythmias, multiorgan failure, where even a small benefit of SDAC may improve outcomes and they consider it a duty of care to administer charcoal. ${ }^{14}$

Even though activated charcoal has been universally used to adsorb a variety of agents and administration of activated charcoal is considered a low-risk intervention, there are substances which are not adsorbed by AC and therefore it should not be used to treat these intoxications. These substances are: hydrocarbons, acids, alkalis, ethanol and heavy metals..$^{1-3,12,14}$ Lapus et al. has used the acronym "PHAILS" to facilitate learning on which intoxications $A C$ is not recommended; note that this is phonetically similar to the word "Fails". ${ }^{23}$ The acronym stands for the following: $P$ is for pesticides, petroleum distillates and unprotected airway. $\mathrm{H}$ is for hydrocarbons, heavy metals and time > th from ingestion. A is for acids, alkali, alcohols and altered level of consciousness with aspiration risk. I is for iron, ileus and intestinal obstruction. $L$ stands for lithium and lack of gag reflex. Finally, $\mathrm{S}$ is for solvents and seizures. ${ }^{23} \mathrm{~A}$ description of all the contraindications for $A C$ are presented in section 5 .

\section{Posology for Single Dose of Activated Charcoal}

The optimal dose of activated charcoal is unknown.' The adsorption can potentially become saturated and therefore with greater area available there is potentially more adsorbed substance. ${ }^{2,10,24}$ The dose most commonly used is a 1:10 ratio of 
Table 2. Recommendations for Use of Multiple Doses of Activated Charcoal.

\begin{tabular}{|c|c|c|c|}
\hline $\begin{array}{c}\text { Indicated } \\
\text { Where elimination was increased. }\end{array}$ & $\begin{array}{l}\text { No support or exclude its use } \\
\text { Studies in Volunteers that demons- } \\
\text { trated increased elimination. }\end{array}$ & $\begin{array}{l}\text { Controversial } \\
\text { Studies are insufficient to recom- } \\
\text { mend its use. }\end{array}$ & $\begin{array}{c}\text { Not recommended } \\
\text { Studies have shown no increase in } \\
\text { elimination. }\end{array}$ \\
\hline Carbamazepine & Amitriptyline & Salicylates & Astemizole \\
\hline Dapsone & Dextropropoxyphene & & Chlorpropamide \\
\hline Quinine & Digoxin & & Imipramine \\
\hline \multirow[t]{3}{*}{ Theophylline } & Disopyramide & & Meprobamate \\
\hline & Nadolol & & Methotrexate \\
\hline & Phenylbutazone & & Phenytoin \\
\hline
\end{tabular}

Adapted from: American Academy of Clinical Toxicology and European Association of Poisons Centres and Clinical Toxicologists. Position Statement and Practice Guidelines on the Use of Multi-Dose Activated Charcoal in the Treatment of Acute Poisoning. Clin Toxicol (Phila). 1999;37(6):731-751. Copyright $\odot ~ 1999$, Taylor U Francis.Reprinted with permission from Taylor $\circlearrowright$ Francis. ${ }^{38}$

drug: charcoal..$^{2,12,24}$

The United States Pharmacopeia (USP DI, 2003) recommended the following oral dosage regimen, which is the dosage recommended by the American Academy of Clinical Toxicology:

- Children up to the first year: 0.5 to $1 \mathrm{~g}$ per $\mathrm{kg} .1$

- Children 1 to 12 years: 0.3 to $1 \mathrm{~g}$ per kg.1

- Adolescents and adults: 25 to $100 \mathrm{~g}$, or $1 \mathrm{~g}$ per kg. The maximum dose is $50 \mathrm{~g}-100 \mathrm{~g} .1,10$

Indications for Administration of Multiple Doses of Activated Charcoal.

Although the administration of multiple doses of activated charcoal has been shown to be beneficial in some cases and may enhance the elimination of a variety of drugs. ${ }^{12,25-37}$ There is no published evidence supporting that administration of multiple doses of activated charcoal results in decreased morbidity or mortality in poisoned patients and therefore routine administration of multiple doses of activated charcoal is not recommended in the poisoned patient.

By administering more than two doses of activated charcoal it is believed that a concentration gradient is maintained in the gastrointestinal tract, and thus as drugs continuously pass through the intestinal bloodstream, these can potentially be absorbed back into the gut and onto the charcoal. This process to enhance elimination is known as "gastrointestinal dialysis". ${ }^{12,17,39,40}$ A summary of the studies performed on AC's enhancement of drug clearance using multiple-dose activated charcoal has been performed and published elsewhere and exceeds the scope of this review. ${ }^{41}$

Gaudreault ${ }^{12}$ suggests to consider administration of multiple doses of activated charcoal if any of the following criteria are met:

1. Intake exceeds the capacity to be adsorbed by a single dose.
2. It can prevent the reabsorption by enterohepatic circulation of the active substance, metabolite, or drug conjugate that is hydrolyzed by bacteria in the bowel.

3. Intoxication by drugs with sustained release.

4. Poisoning by drugs that decrease gastrointestinal transit (anticholinergics, tricyclic antidepressants, opioids, and phenothiazine).

The list of toxins on which clearance can potentially be increased with multiple doses of activated charcoal are described in Table $1 .{ }^{12}$ In the position paper by the American Academy of Clinical Toxicology and the European Association of Poisons Centres and Clinical Toxicologists the recommendation is that administration of multiple doses of activated charcoal should be considered in intoxications where there has been a life threatening dose of carbamazepine, dapsone, phenobarbital, quinine, or theophylline ingested as this could potentially avoid the need for invasive extracorporeal techniques needed for treatment, but defer the ultimate decision on clinical judgement by the treating physician, presence of contraindications and effectiveness of other methods of treatment. ${ }^{3}$ Their recommendations for use of multiple doses of activated charcoal are illustrated in Table $2 . .^{38}$

\section{Posology for Multiple Doses of Activated Charcoal:}

More studies are needed to identify the optimal dose for administration of multiple doses of activated charcoal. ${ }^{38}$ The recommendation is to administer from 0.25 to $1 \mathrm{~g} / \mathrm{kg}$ every 1-4 hours, when there is clinical evidence of absorption of the substance either by laboratory tests (increasing serum concentration) or known nature of the substance (sustained release formulations). ${ }^{12}$ Doses of activated charcoal should be continued until the patient's condition improves by clinical and/or laboratory evidence. ${ }^{12}$ One of the major limitations of multiple dosing is increasing nausea or emesis. This could be improved by administrating lower doses in shorter time intervals or continuous administration through a nasogastric tube. In some cases its necessary to initiate antiemetics. ${ }^{12,38}$ 


\section{Contraindications}

Contraindications to the Use of a Single Dose of Activated Charcoal

- Use in the setting of intoxication by substances not adsorbed by $A C$ (see section 4.1)

- Substances that have a specific antidote: Administration of the antidote should be preferred because concurrent administration of activated charcoal with the antidote can reduce the effect of the antidote. ${ }^{2,3}$

- Non-intact gastrointestinal tract: Patients with anatomic or functional abnormalities of the gastrointestinal tract. For example, patients with intestinal obstruction.

- Patient with an unprotected airway or with altered mental status without endotracheal intubation..$^{1,3,11,12}$ Consider that some patients can compromise their airway if the toxic agent is a central nervous system depressant. ${ }^{2}$

- If their use increases the risk of aspiration: For example poisoning by a hydrocarbon with high aspiration potential. ${ }^{1-2,3,11}$

- Pathologies, recent surgeries or medical conditions that increase the risk of gastrointestinal bleeding or perforation. $^{1-2,3}$

- Use on intoxication by corrosive substances. It's not an absolute contraindication, can be considered if the dose and agent ingested can potentially cause systemic toxicity. ${ }^{1,3}$

Contraindications to the use of multiple doses of activated charcoal

The recommendations from the American Academy of Clinical Toxicology and the European Association of Poisons Centres and Clinical Toxicologists are listed below. ${ }^{38}$

Absolute contraindications to the use of MDAC are:

- An unconscious patient with an unprotected airway

- Presence of intestinal obstruction

- An injured gastrointestinal tract

Relative contraindications to the use of MDAC are:

- Decreased peristalsis

Consider that the patient has decreased peristalsis if there are decreased bowel sounds, abdominal distention or ileus. For example in cases of opioid overdose or anticholinergics. If activated charcoal is administered then the physician should monitor for gastrointestinal obstruction and prevention of aspiration. $^{38}$

\section{Use of Activated Charcoal in the Emergency Department Initial management}

When the patient arrives at the emergency room the initial resuscitation must be performed immediately. Evaluation of the intoxicated patient should start with initial supportive measures and management of the Circulation, Airway, Breathing (CAB's), a history and physical examination, toxidrome recognition, diagnostic testing and then start considering whether or not to administer decontamination measures, enhanced elimination measures or antidotes and consult toxicologists or regional poison control center. ${ }^{42,5,43}$

Subsequently, the physician should assess the risks, consider the possible clinical course and potential complications for the patient, as well as the risks and benefits of giving therapies to prevent absorption, increase elimination or indications for antidotes. Therefore, the decision to give a patient activated charcoal should be undertaken using the principles of evidence-based medicine where the clinician should make a value-based judgement on each individual patient. ${ }^{14,44}$ Although there is little controlled trial evidence to support the administration of activated charcoal, the quantitative studies and evidence in small studies of select drugs suggest there is potential benefit in some situations and therefore it can have a significant role in an individual patient. ${ }^{14,44}$

Some authors suggest that the preferred method for gastrointestinal decontamination in awake patients with intact airway is AC. ${ }^{5}$ For this process one must consider: the agent, dose, time from ingestion, current clinical presentation and individual patient factors. This process involves factors specific to the physician (experience, knowledge) and the patient (medical conditions, information provided). ${ }^{3,45}$ Bailey proposed the decontamination triangle as a tool to help clinicians decide on administration of $A C$ or not based on the potential toxicity of the poison, the benefit of $A C$, balanced against the risks of charcoal.3,22 Activated charcoal or any other method of gastrointestinal decontamination should only be considered if there has been ingestion of a potentially toxic amount of substance that is severe and/or can threaten the patient's life and supportive management or antidotes may not be sufficient to ensure an optimal result. Some authors have recommended against use in mild to moderate intoxications. ${ }^{11}$

\section{Administration of Activated Charcoal by Nasogastric Tube}

The following description for administration of AC was adjusted from the "Protocol for Management of the Intoxicated Patient, Measures of Gastrointestinal Decontamination: Activated Charcoal" from the Hospital Universitario del Valle, in Cali, Colombia, written by JBV and Dr. Maurix Rojas, used with permission.

1. Put the patient in a comfortable position, preferably in sitting or left lateral position with the head at 450 angle and explain the procedure.

2. Isolate the airway with an endotracheal tube in patients with altered consciousness.

3. The size of the catheter will depend on the size and appearance of the patient: Adults can usually start with a 16 to $20 \mathrm{Fr}$ catheter.

4. Measure the distance between the earlobe, the nasal apex and from there to the xiphoid to estimate the total length of the catheter that should be introduced.

5. Quickly and carefully insert the catheter in the nose. Lubricate the catheter with water prior to insertion.

6. Check that the catheter is in the stomach by flushing air with a syringe at the proximal end of the catheter and simultaneously auscultating on the epigastrium.

7. Secure the catheter to prevent movement during the procedure

8. Perform gastric lavage according to the existing protocol at your institution.

9. Prepare a solution of activated charcoal in a ratio of 1 gram of activated charcoal per $5 \mathrm{ml}$ of distilled water (1:5).

10. Flush the catheter by administering $20 \mathrm{cc}$ of distilled water or saline. 
11. Dispense the total dose of activated charcoal with a $50 \mathrm{cc}$ syringe through the catheter quickly.

12. Flush the catheter by administering $20 \mathrm{cc}$ of distilled water or saline

13. Remove the nasogastric catheter immediately after the procedure.

14. Situate the patient in supine position with the head of bed elevated to $45^{\circ}$.

15. Record of the procedure in the medical record.

The clinical use of presentations of activated charcoal in tablets or capsules or super-activated charcoal is not recommended. ${ }^{1,10}$

\section{Complications}

Administration of activated charcoal is considered generally well tolerated, these are the reported adverse reactions and side effects.

- Aspiration: Some cases have been reported where patients aspirated gastric contents and the clinical picture has ranged from developing pneumonitis to rapid-onset adult respiratory distress syndrome and death. ${ }^{11,46-48} \mathrm{~A}$ randomized clinical trial found equal rate of aspiration pneumonia among the group receiving supportive care and those who received activated charcoal..$^{20}$ While another large study of overdose patients looking at risk factors for aspiration pneumonitis did not identify activated charcoal as a risk factor. ${ }^{46}$ Therefore the true effect is currently unknown.

- Emesis: Is generally considered an infrequent but the most common side effect.20 Reports have ranged from about $7 \%$ to $15 \%$ and even $25 \%$ of patients can have emesis although the trial by Cooper et al reported no difference in emesis when compared to patients who did not receive activated charcoal. 14,20,49

- Taste: Ingestion of activated charcoal is reported to be distasteful to drink which may cause patient dissatisfaction. ${ }^{14}$

- Gastrointestinal obstruction: Has been reported mainly with use of multiple doses of activated charcoal but some studies have reported an incidence of $0 \% .48$ In the literature there are 9 case reports of intestinal obstruction since $1981 .{ }^{48}$

- Constipation. More frequently observed with multiple doses. 1,38

- Diarrhea.

- Gastrointestinal perforation: There have been a few cases reported with use of multiple dose activated charcoal or after nasogastric tube placement. ${ }^{38,50}$

- Electrolyte disturbances. Mainly related to use of multiple doses of activated charcoal. ${ }^{38}$ One study found an incidence of hypernatremia 1: 176 patients ( $>155 \mathrm{mEq} / \mathrm{L}$ ), and hypermagnesemia in 1: 292 patients $(33.75 \mathrm{mEq} / \mathrm{L}) .^{48}$

- Death: Although very rare, in some case reports have found no cause of death from poisoning but activated charcoal. 1,38 


\section{References}

1. American Academy of Clinical Toxicology, European Association of Poisons Centres and Clinical Toxicologists. Position Paper: Single-Dose Activated Charcoal. Clinical Toxicology. 2005;43(2):61-87.

2. Greene S, Harris C, Singer J. Gastrointestinal decontamination of the poisoned patient. Pediatr Emerg Care. Mar 2008;24(3):176-186; quiz 187-179.

3. Bailey B. To Decontaminate or Not to Decontaminate? The Balance Between Potential Risks and Foreseeable Benefits. Clin Pediatr Emerg Med. 2008;9(1):17-23.

4. Mowry JB, Spyker DA, Brooks DE, McMillan N, Schauben JL. 2014 Annual Report of the American Association of Poison Control Centers' National Poison Data System (NPDS): 32nd Annual Report. Clin Toxicol (Phila). 2015;53(10):962-1147.

5. Thompson TM, Theobald J, Lu J, Erickson TB. The general approach to the poisoned patient. Dis Mon. Nov 2014;60(11):509524.

6. Pruss-Ustun A, Vickers C, Haefliger P, Bertollini R. Knowns and unknowns on burden of disease due to chemicals: a systematic review. Environ Health. Jan 21 2011;10:9.

7. Bergen $\mathrm{G}$, Chen $\mathrm{LH}$, Warner M, Fingerhut LA. Injury in the United States: 2007 Chartbook. Hyattsville, MD:. National Center for Health Statistics. 2008.

8. CDC. Increases in age-group-specific injury mortality - United States, 1999-2004. MMWR. 2007;56:1281-1284.

9. Cooney DO. A "superactive" charcoal for antidotal use in poisonings. Clin Toxicol. 1977;11(4):387-390.

10. Heard K. Gastrointestinal decontamination. Med Clin North Am. Nov 2005;89(6):1067-1078.

11. Seger D. Single-dose activated charcoal-backup and reassess. J Toxicol Clin Toxicol. 2004;42(1):101-110.

12. Gaudreault P. Activated Charcoal Revisited. Clin Ped Emerg Med 2005;6:76-80.

13. Jurgens $G$, Hoegberg LC, Graudal NA. The effect of activated charcoal on drug exposure in healthy volunteers: a meta-analysis. Clin Pharmacol Ther. May 2009;85(5):501-505.

14. Isbister GK, Kumar VV. Indications for single-dose activated charcoal administration in acute overdose. Curr Opin Crit Care. Aug 2011;17(4):351-357.

15. Chyka PA, Seger D. Position statement: single-dose activated charcoal. American Academy of Clinical Toxicology; European Association of Poisons Centres and Clinical Toxicologists. J Toxicol Clin Toxicol. 1997;35(7):721-741.

16. Cooper GM, Le Couteur DG, Richardson D, Buckley NA. A randomized clinical trial of activated charcoal for the routine management of oral drug overdose. QJM. Sep 2005;98(9):655-660. 17. Eddleston M, Juszczak E, Buckley NA, et al. Multiple-dose activated charcoal in acute self-poisoning: a randomised controlled trial. Lancet. Feb 16 2008;371(9612):579-587.

18. Olson KR. Activated charcoal for acute poisoning: one toxicologist's journey. J Med Toxicol. Jun 2010;6(2):190-198.

19. Bond GR. The role of activated charcoal and gastric emptying in gastrointestinal decontamination: a state-of-the-art review. Ann Emerg Med. Mar 2002;39(3):273-286.

20. Merigian KS, Blaho KE. Single-dose oral activated charcoal in the treatment of the self-poisoned patient: a prospective, randomized, controlled trial. Am J Ther. Jul-Aug 2002;9(4):301-308. 21. Chiew AL, Fountain JS, Graudins A, Isbister GK, Reith D, Buckley NA. Summary statement: new guidelines for the management of paracetamol poisoning in Australia and New Zealand.
Med J Aust. Sep 7 2015;203(5):215-218.

22. Bailey B. Gastrointestinal decontamination triangle. Clin Toxicol (Phila). 2005;43(1):59-60.

23. Lapus RM. Activated charcoal for pediatric poisonings: the universal antidote? Curr Opin Pediatr. Apr 2007;19(2):216-222.

24. Heard K. The changing indications of gastrointestinal decontamination in poisonings. Clin Lab Med. Mar 2006;26(1):1-12, vii.

25. de Silva HA, Fonseka MM, Pathmeswaran A, et al. Multiple-dose activated charcoal for treatment of yellow oleander poisoning: a single-blind, randomised, placebo-controlled trial. Lancet. Jun 7 2003;361(9373):1935-1938.

26. Mahutte CK, True RJ, Michiels TM, Berman JM, Light RW. Increased serum theophylline clearance with orally administered activated charcoal. Am Rev Respir Dis. Nov 1983;128(5):820-822. 27. Pond S, Jacobs M, Marks J, Garner J, Goldschlager N, Hansen D. Treatment of digitoxin overdose with oral activated charcoal. Lancet. Nov 21 1981;2(8256):1177-1178.

28. Pond SM, Olson KR, Osterloh JD, Tong TG. Randomized study of the treatment of phenobarbital overdose with repeated doses of activated charcoal. JAMA. Jun 15 1984;251(23):3104-3108. 29. Neuvonen PJ, Elonen E, Mattila MJ. Oral activated charcoal and dapsone elimination. Clin Pharmacol Ther. Jun 1980;27(6):823-827.

30. Berg MJ, Berlinger WG, Goldberg MJ, Spector R, Johnson GF. Acceleration of the body clearance of phenobarbital by oral activated charcoal. N Engl J Med. Sep 9 1982;307(11):642-644. 31. du Souich P, Caille $G$, Larochelle P. Enhancement of nadolol elimination by activated charcoal and antibiotics. Clin Pharmacol Ther. May 1983;33(5):585-590.

32. Berlinger WG, Spector R, Goldberg MJ, Johnson GF, Quee CK, Berg MJ. Enhancement of theophylline clearance by oral activated charcoal. Clin Pharmacol Ther. Mar 1983;33(3):351-354. 33. Hillman RJ, Prescott LF. Treatment of salicylate poisoning with repeated oral charcoal. Br Med J (Clin Res Ed). Nov 23 1985;291(6507):1472.

34. Honcharik N, Anthone S. Activated charcoal in acute cyclosporin overdose. Lancet. May 4 1985; (8436):1051.

35. Karkkainen S, Neuvonen PJ. Effect of oral charcoal and urine pH on dextropropoxyphene pharmacokinetics. Int J Clin Pharmacol Ther Toxicol. Apr 1985;23(4):219-225.

36. Neuvonen PJ, Elonen E. Effect of activated charcoal on absorption and elimination of phenobarbitone, carbamazepine and phenylbutazone in man. Eur J Clin Pharmacol. Jan 1980;17(1):51-57.

37. Chan BS, Sellors K, Chiew AL, Buckley NA. Use of multi-dose activated charcoal in phenytoin toxicity secondary to genetic polymorphism. Clin Toxicol (Phila). Feb 2015;53(2):131-133.

38. Toxicology; AAOC, Toxicologists EAoPCaC. Position statement and practice guidelines on the use of multi-dose activated charcoal in the treatment of acute poisoning. I Toxicol Clin Toxicol. 1999;37(6):731-751.

39. Tenenbein M. Multiple doses of activated charcoal: time for reappraisal II. Ann Emerg Med. Oct 2003;42(4):597-598; author reply 598-599.

40. Levy G. Gastrointestinal clearance of drugs with activated charcoal. N Engl J Med. Sep 9 1982;307(11):676-678.

41. Chyka PA. Multiple-dose activated charcoal and enhancement of systemic drug clearance: summary of studies in animals and human volunteers. J Toxicol Clin Toxicol. 1995;33(5):399-405. 42. Mokhlesi B, Leiken JB, Murray P, Corbridge TC. Adult toxico- 
logy in critical care: part I: general approach to the intoxicated patient. Chest. Feb 2003;123(2):577-592.

43. Ocal 0, Ozucelik DN, Avci A, et al. A comparison of the outcome of CPR according to AHA 2005 ACLS and AHA 2010 ACLS guidelines in cardiac arrest: multicenter study. Int J Clin Exp Med. 2015;8(11):21549-21556.

44. Shin JJ, Randolph GW, Rauch SD. Evidence-based medicine in otolaryngology, part 1: the multiple faces of evidence-based medicine. Otolaryngol Head Neck Surg. May 2010;142(5):637646.

45. Daly FF, Little M, Murray L. A risk assessment based approach to the management of acute poisoning. Emerg Med J. May 2006;23(5):396-399.

46. Isbister CK, Downes F, Sibbritt D, Dawson AH, Whyte IM. Aspiration pneumonitis in an overdose population: frequency, predictors, and outcomes. Crit Care Med. Jan 2004;32(1):88-93. 47. De Weerdt A, Snoeckx A, Germonpre P, Jorens PG. Rapid-onset adult respiratory distress syndrome after activated charcoal aspiration. A pitch-black tale of a potential to kill. Am J Respir Crit Care Med. Feb 1 2015;191(3):344-345.

48. Dorrington CL, Johnson DW, Brant R, Multiple Dose Activated Charcoal Complication Study G. The frequency of complications associated with the use of multiple-dose activated charcoal. Ann Emerg Med. Mar 2003;41(3):370-377.

49. Orfanidou G, Chalkias A, Koutsovasilis A, Loizos G, Xanthos T. Activated charcoal may not be necessary in all oral overdoses of medication. Am J Emerg Med. Feb 2016;34(2):319-321.

50. Lobo-Machin I, Medina-Arana V, Delgado-Plasencia L, Bravo-Gutierrez A, Burillo-Putze G. Activated carbon peritonitis. Cir Esp. Nov 2015;93(9):e107-109.

\section{Acknowledgment}

The authors would like to acknowledge Dr. Maurix Fernando Rojas for sharing his vast knowledge and experience in the area of toxicology and his support during the early planning phase of this manuscript.

\section{Conflict of Interest Statement it Funding}

None of the authors shows any conflicts of interest during the investigation or publication of this article.

\section{Author Contributions}

Conception and design the work/idea: JBV. Analysis and interpretation of data, write the manuscript: JBV, DJ. Critical revision of the manuscript: JBV. Approval of the final version, administrative of technical advice: JBV, DJ.

Cite as:

Bonilla-Velez J, Marin-Cuero DJ. The Use of Activated Charcoal for Acute Poisonings. Int J Med Students. 2017 Jan-Apr;5(1):45-52. 